\title{
Effects of a postnatal Atrx conditional knockout in neurons on autism-like behaviours in male and female mice
}

\author{
Nicole Martin-Kenny ${ }^{1,2,3}$ and Nathalie G. Bérubé ${ }^{1,2,3,4^{*}}$ (D)
}

\begin{abstract}
Background: Alpha-thalassemia/mental retardation, X-linked, or ATRX, is an autism susceptibility gene that encodes a chromatin remodeler. Mutations of ATRX result in the ATR-X intellectual disability syndrome and have been identified in autism spectrum disorder (ASD) patients. The mechanisms by which ATRX mutations lead to autism and autistic-like behaviours are not yet known. To address this question, we generated mice with postnatal Atrx inactivation in excitatory neurons of the forebrain and performed a battery of behavioural assays that assess autistic-like behaviours.

Methods: Male and female mice with a postnatal conditional ablation of ATRX were generated using the Cre/lox system under the control of the aCaMKIl gene promoter. These mice were tested in a battery of behavioural tests that assess autistic-like features. We utilized paradigms that measure social behaviour, repetitive, and stereotyped behaviours, as well as sensory gating. Statistics were calculated by two-way repeated measures ANOVA with Sidak's multiple comparison test or unpaired Student's $t$ tests as indicated.
\end{abstract}

Results: The behaviour tests revealed no significant differences between Atrx-cKO and control mice. We identified sexually dimorphic changes in odor habituation and discrimination; however, these changes did not correlate with social deficits.

Conclusion: The postnatal knockout of Atrx in forebrain excitatory neurons does not lead to autism-related behaviours in male or female mice.

Keywords: Autism spectrum disorder, ATRX, Social behaviours, Repetitive behaviours, Startle response, Genetically engineered mice, Cre/loxP system

\section{Background}

Autism spectrum disorder (ASD) is a behaviourally defined condition characterized by deficits in social and communicative abilities, impaired sensory gating, as well as the presence of stereotyped behaviours $[1,2]$. Recent work has highlighted the important contribution of de novo variants and inherited copy number variants in ASD, confirming a strong genetic component of this disease [1-3]. Numerous

\footnotetext{
* Correspondence: nberube@uwo.ca

'Department of Paediatrics, Schulich School of Medicine and Dentistry, Western University, London, Ontario, Canada

${ }^{2}$ Department of Anatomy and Cell Biology, Schulich School of Medicine and Dentistry, Western University, London, Ontario, Canada

Full list of author information is available at the end of the article
}

autism susceptibility genes have been identified and shown to share commonalities in synaptic, transcriptional, and epigenetic mechanisms [4-6]. Mouse models have typically been used to investigate the behavioural implications of genetic mutations associated with ASD [7, 8]. However, these studies often omit the investigation of the sex-specific effects of these genetic mutations, limiting the potential translational applications. In the general population, ASD occurs at a 4:1 male:female ratio, highlighting the need to study the outcome of genetic mutations in both male and female model systems $[1,2]$.

In this study, we describe the impact of targeted inactivation of Atrx in glutamatergic neurons on behaviours 
related to autism in male and female mice. ATRX belongs to the SWI/SNF family of chromatin remodeling factors $[9,10]$. Mutations in the ATRX gene are associated with an intellectual disability syndrome referred to as ATRXsyndrome, characterized by autistic-like behaviours in addition to cognitive deficits, intellectual disabilities, and developmental delays [11]. Furthermore, autistic carriers of rare mutations in ATRX have been discovered and missense variants in $A T R X$ have been identified in male ASD patients. Interestingly, female carriers of ATRX mutations experience skewed $\mathrm{X}$-inactivation, and as a result, are asymptomatic [12-18].

Previous studies have demonstrated that the loss of Atrx in the mouse forebrain causes changes in gene expression $[19$, 20]. Specifically, transcription of autism susceptibility genes, including the monogenic Neuroligin-4 (Nlgn4), are altered upon loss of Atrx [19]. Additionally, sexually dimorphic transcript changes have been revealed in the adult mouse hippocampus upon the loss of Atrx in excitatory neurons [21]. However, autistic behaviours were not evaluated in that report and should be addressed given the link between ATRX mutations and ASD.

In this study, we characterize the impact of Atrx loss in neurons on autistic-like behaviours in male and female mice. We used the AtrxCamKIICre model [21] where Atrx is ablated in forebrain excitatory neurons postnatally, thus bypassing deleterious effects of ATRX loss-of-function previously observed in neural progenitors during brain development $[22,23]$. An array of behavioural assays was performed to investigate the presence of autistic-like behaviours, including deficits in sociability, altered sensory gating, and the presence of repetitive or stereotyped behaviours. These investigations revealed minimal behavioural deficits related to autism in both male and female mice. Interestingly, we identified changes in olfaction, particularly odor discrimination, in both male and female mice upon the conditional loss of Atrx in neurons. Overall, this study demonstrates that a conditional loss of Atrx in forebrain excitatory neurons postnatally does not result in typical autistic-like traits in male or female mice.

\section{Materials and methods}

\section{Animal care and husbandry}

Mice were exposed to a 12-h light/12-h dark cycle and with water and chow ad libitum. The Atrxx ${ }^{\operatorname{loxP}}$ females (129/Sv background) have been described previously [23]. Atrx ${ }^{\text {loxP }}$ mice were mated with C57BL/6 mice expressing Cre recombinase under the control of the $\alpha$ CaMKII gene promoter [24]. The progeny includes hemizygous male mice that produce no ATRX protein in forebrain excitatory neurons $\left(\operatorname{Atr} x\right.$-cKO $\left.{ }^{\mathrm{MALE}}\right)$. The Atrx-cKO males were mated to Atr $x^{\text {loxP }}$ females to yield homozygous deletion of Atrx in female mice (Atrx-cKO $\left.{ }^{\text {FEMALE }}\right)$. Male and female littermate floxed mice lacking the Cre allele were used as controls $\left(\mathrm{Ctrl}^{\mathrm{MALE}} ; \mathrm{Ctrl}^{\mathrm{FEMALE}}\right)$. Consequently, male and female mice are from different hybrid generations. Control littermates from the same hybrid generation as the corresponding conditional knockout mice were used for behavioural assays. Genotyping of tail biopsies for the presence of the floxed and Cre alleles was performed as described previously [23]. Conditional loss of the ATRX protein in postnatal neurons was previously verified in adult Atrx$\mathrm{cKO}^{\mathrm{MALE}}$ and Atrx-cKO ${ }^{\mathrm{FEMALE}}$ forebrain [21]. All procedures involving animals were conducted in accordance with the regulations of the Animals for Research Act of the province of Ontario and approved by the University of Western Ontario Animal Care and Use Committee (2017048). Behavioural assessments started with less demanding tasks and moved to more demanding tasks in the following order: open-field test, marble-burying assay, induced self-grooming, pre-pulse inhibition (PPI) and startle response, social approach, and 3-chamber social tests. ARRIVE guidelines were followed: mouse groups were randomized, experimenters were blind to the genotypes, and software-based analysis was used to score mouse performance in all the tasks. All behavioural tasks were performed between 9:00 AM and 4:00 PM. All behavioural assays were performed when mice were between 3 and 7 months of age. Three cohorts of male and female mice were used to reach the final sample size $\left(\mathrm{Ctrl}^{\mathrm{MALE}}: 17\right.$; Atr $x$-cKO ${ }^{\text {MALE }}: 10$; Ctrl $^{\text {FEMALE }}: 13$; Atr $x$-cKO ${ }^{\text {FEMALE }}: 13$ ). Statistics were calculated by two-way repeated measures ANOVA with Sidak's multiple comparison test or unpaired Student's $t$ tests, as indicated in the figure legends.

\section{Odor habituation and discrimination}

The odor habituation and discrimination assay was performed as previously described [25] to assess olfaction. Individual mice were placed into a clean cage with a wire lid and allowed to habituate to the testing room for $30 \mathrm{~min}$. The mice were then presented with an odor on a cotton swab (either almond, banana, or water as a control) for a 2 -min trial. For each trial, $50 \mu \mathrm{l}$ of water, almond extract, or banana extract (club house) was pipetted onto the tip of a cotton swab and the swab was then secured to the wire cage top through the water bottle opening. The mice were presented with the same odor three times before being presented with a new odour, for a total of nine trials. During the 2-min trials, the amount of time that the mouse spent sniffing the odor was recorded by an investigator blind to the genotype. Sniffing was defined as the animal's nose being in proximity to the cotton swab $(2 \mathrm{~cm}$ or closer), and oriented toward the swab.

\section{Social approach}

This test was performed as previously described [26] to assess for sociability with conspecific mice. For two 
consecutive days prior to the test day, individual mice were habituated to the open area for $10 \mathrm{~min}$. On the test day, pairs of unfamiliar, same-sex conspecific mice were placed into the cage. Behaviour of the mice was recorded by the AnyMaze software and video-tracking system. The time spent in social interaction, defined as the experimental mouse sniffing the stranger mouse, was manually scored by investigators unaware of the genotype.

Three-chamber social tests (social preference and novelty) The social preference and social novelty assessments were performed as described $[26,27]$ with minor modifications. Individual mice were placed in the 3-chambered box and allowed to freely explore the arena during a 10min habituation period. After the habituation period, an unfamiliar, same-sex mouse of a different genotype (stranger 1) was placed in one of the side chambers under a wire cage. An identical wire cage containing an inanimate object was placed in the opposite chamber. The test mouse was then allowed to explore the entire 3 -chambered arena for $10 \mathrm{~min}$. The amount of time spent in each chamber was recorded by the AnyMaze video-tracking system. Following this period, a second unfamiliar, same-sex mouse of a different genotype (stranger 2) was placed into the wire cage previously containing the inanimate object. The test mouse was then allowed to explore the 3-chambered arena for $10 \mathrm{~min}$. The amount of time spent in each chamber was recorded by the AnyMaze video-tracking system. Based on the amount of time spent in each chamber, a 'sociability index' and a 'social novelty index' was calculated as previously described [27]. The sociability index was calculated as time stranger $/$ (time $_{\text {stranger }}+$ time $\left._{\text {object }}\right) \times 100$. The social novelty index was calculated as time $_{\text {novel }} /\left(\right.$ time $_{\text {novel }}+$ time $\left._{\text {familiar }}\right) \times 100$.

\section{Marble burying}

The test was performed as previously described [28] with modifications to evaluate repetitive digging behaviour. Mice were brought into the test room to habituate in their home cages for approximately $30 \mathrm{~min}$ prior to the test. The test cages were filled with $4 \mathrm{~cm}$ of wood-chip bedding, with 12 evenly spaced glass marbles placed on the surface. Individual mice were then placed in the test cage and permitted to explore for $30 \mathrm{~min}$. Following the test, the number of marbles buried ( $>3 / 4$ surface covered) was counted and recorded by investigators blind to the genotype.

\section{Induced self-grooming}

The test was performed as previously described [27, 29] to evaluate repetitive grooming tendencies. Mice were individually habituated in an empty test cage for $30 \mathrm{~min}$ prior to the test. To amplify natural grooming tendencies, mice were misted with water 3 times at $10 \mathrm{~cm}$ distance of the upper-back. Following this misting, the grooming behaviour of each mouse was recorded by the Anymaze video-tracking system for $30 \mathrm{~min}$. The time that each individual mouse spent grooming during this 30-min trial was manually scored by the rater, unaware of the genotype.

\section{Open-field test}

Mice were brought into the testing room to habituate in their home cages approximately $30 \mathrm{~min}$ prior to the test. Mice were placed in a $20 \mathrm{~cm} \times 20 \mathrm{~cm}$ arena with $30 \mathrm{~cm}$ high walls. Locomotor activity was automatically recorded in 5-min intervals over $2 \mathrm{~h}$ (AccuScanInstrument) [30]. For each mouse the number of vertical episodes was assessed.

\section{Pre-pulse inhibition of the startle response}

The pre-pulse inhibition and startle response tests were performed as previously described [31] to assess sensory gating. Mice underwent two days of habituation prior to the testing day, to acclimate the mice to the apparatus. During this habituation, mice were individually placed in the chamber apparatus and exposed to background noise (65 db) for 5 min (SR-LAB, San Diego Instruments). On the test day, individual mice were placed in the chamber and acclimated for $10 \mathrm{~min}$ with background noise. The mice then underwent a habituation block, consisting of 50 acoustic startle trials, with 20 ms stimulus of $115 \mathrm{db}$, and intertrial interval of $20 \mathrm{~s}$. After the habituation block, mice underwent a prepulse-inhibition block consisting of ten sets of five types of trials randomly ordered with variable intertrial intervals of 10,15 , or $20 \mathrm{~s}$. Four of the five trial types consisted of prepulses (intensity of 75 or $80 \mathrm{db}$, length of $20 \mathrm{~ms}$ ), separated from the startle stimulus (intensity of $115 \mathrm{db}$, length of $40 \mathrm{~ms}$ ) by an interstimulus interval of either $30 \mathrm{~ms}$ or $100 \mathrm{~ms}$. The fifth trial type was a startle pulse alone. The startle response was measured by the movement of the mouse on the platform, which generates a transient force analyzed by the software. The startle magnitude recorded was an average for the ten trials of each trial type and startle magnitudes of pre-pulse trials were normalized to the pulse-only trial.

\section{Results}

Sexually dimorphic olfaction differences in Atrx-cKO mice As olfactory impairments can confound the interpretation of other tests, especially social behaviour assays, we first wanted to address whether the loss of Atrx in excitatory neurons of the forebrain alters olfaction in male and female mice. To do this, we performed the odor discrimination and habituation assay [25]. In this test, mice were presented with multiple odors for 2-min trials, during which the amount of time spent sniffing the odor was recorded. During this test, Atrx-cKO ${ }^{\mathrm{MALE}}$ 
mice spent significantly less time sniffing the odors throughout the nine trials compared to $\mathrm{Ctrl}^{\mathrm{MALE}}$ mice (ANOVA, ${ }^{* *} p=0.004$; Fig. 1a). In particular, Atrx-cKOMALE mice spent significantly less time sniffing the cotton swab when first presented with the banana odor (multiple comparisons, ${ }^{* * * *} p<0.001$ ). There was no significant difference in the overall amount of time spent sniffing the odors throughout the test between AtrxcKO $^{\text {FEMALE }}$ and $\mathrm{Ctrl}^{\text {FEMALE }}$ mice. However, Atr $x$-cKO ${ }^{\mathrm{FE}-}$ MALE mice did spend significantly more time sniffing the cotton swab when first presented with the banana odor (multiple comparison, ${ }^{* * * * *} p<0.0001$; Fig. 1b). Overall, the results of this test suggest that the loss of Atrx in forebrain excitatory neurons results in sexually dimorphic changes in olfaction that must be considered in subsequent behaviour testing of these mice.

\section{Social assays reveal no deficits in Atrx-CKO ${ }^{\text {MALE }}$ and Atrx- CKO ${ }^{\text {FEMALE }}$ mice}

Given that ATRX mutations are associated with autistic traits in humans, we next sought to investigate if the loss of Atrx in forebrain excitatory neurons has an effect on social behaviour. Changes in sociability and social preference are some of the most common deficits observed in mouse models with autism-associated genetic mutations [26, 27, 32-34]. As such, we first investigated sociability of Atrx-cKO ${ }^{\text {MALE }}$ and Atrx-cKO ${ }^{\mathrm{FEMALE}}$ mice by means of the social approach assay, as described previously [26]. There was no significant difference in the total amount of time that Atrx-cKO ${ }^{\text {MALE }}$ and Atrx-cKOFEMALE mice spent interacting with a stranger mouse compared to controls (Fig. 2a). However, when these results were grouped and analyzed by sex, male mice (Atrx-cKO ${ }^{\text {MALE }}$ and $\mathrm{Ctrl}^{\mathrm{MALE}}$ ) spent more time socially interacting with the stranger mouse compared to female mice (Atr $x$-cKO ${ }^{\mathrm{FEMALE}}$ and $\mathrm{Ctrl}^{\mathrm{FEMALE}}$ ) (ANOVA, " $p=$ 0.048; Fig. 2a). When social interaction was analyzed over 1-min intervals during the 10-min test, there were no genotypic or sex-differences (Fig. 2b).

We also investigated social preference and social novelty in the three-chambered paradigm [27]. During the first part of the paradigm, social preference was assessed as mice were placed into a three-chambered apparatus and were free to explore between the chambers. The outer two chambers contained either a novel object or a stranger mouse, while the centre chamber remained empty. Both $\mathrm{Ctrl}^{\mathrm{MALE}}$ and Atrx-cKO ${ }^{\mathrm{MALE}}$ mice demonstrated a preference for the chamber containing a stranger mouse compared to the object and the empty chamber (multiple comparisons, ${ }^{* * * *} p<0.0001$; Fig. 2c). Similarly, both $\mathrm{Ctrl}^{\mathrm{FEMALE}}$ and Atrx-cKO ${ }^{\mathrm{FEMALE}}$ mice preferred exploration of the stranger mouse (multiple comparisons, ${ }^{*} p=0.030,{ }^{* * * * *} p<0.0001$; Fig. $2 \mathrm{~d}$ ). No genotypic differences were observed in social preference between groups (Fig. 2c, d).

Social novelty was investigated during the second part of the paradigm in which the outer chambers contained either the stranger mouse from the first part of the test (familiar mouse) or a novel mouse. Ctrl ${ }^{\mathrm{MALE}}$ and Atrx$\mathrm{CKO}^{\mathrm{MALE}}$ mice both spent more time in the chamber containing the novel mouse compared to the familiar mouse and the empty chamber (multiple comparisons, $* * * * 0.001,{ }^{* * * * * *} p<0.0001$; Fig. 2e). Interestingly, although $\mathrm{Ctrl}^{\text {FEMALE }}$ and Atrx-cKO ${ }^{\text {FEMALE }}$ mice both spent significantly less time in the empty chamber, neither demonstrated a preference for the novel mouse over the familiar mouse (multiple comparisons, ${ }^{* * *} p<0.001$; Fig. 2f). Sociability and social memory indexes were

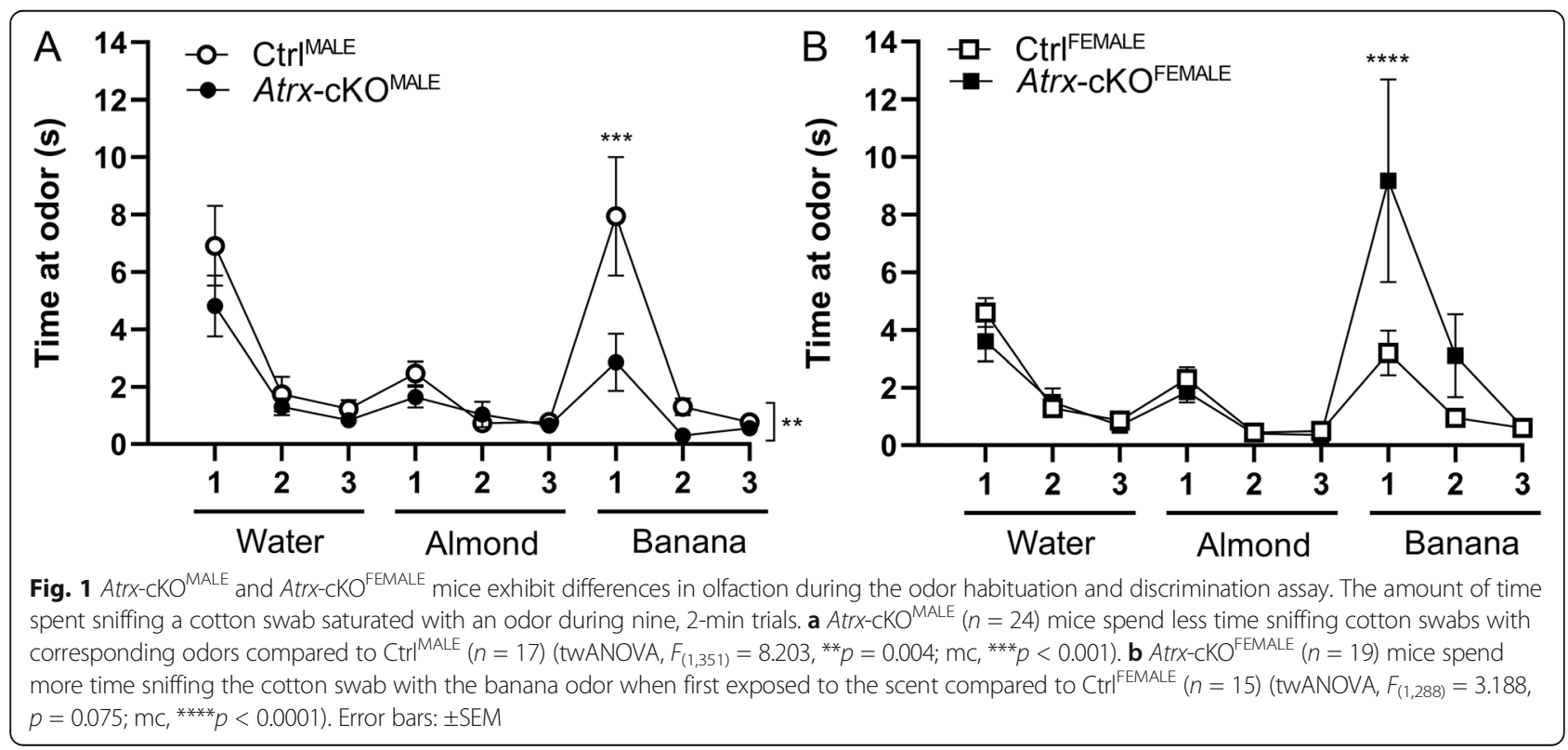




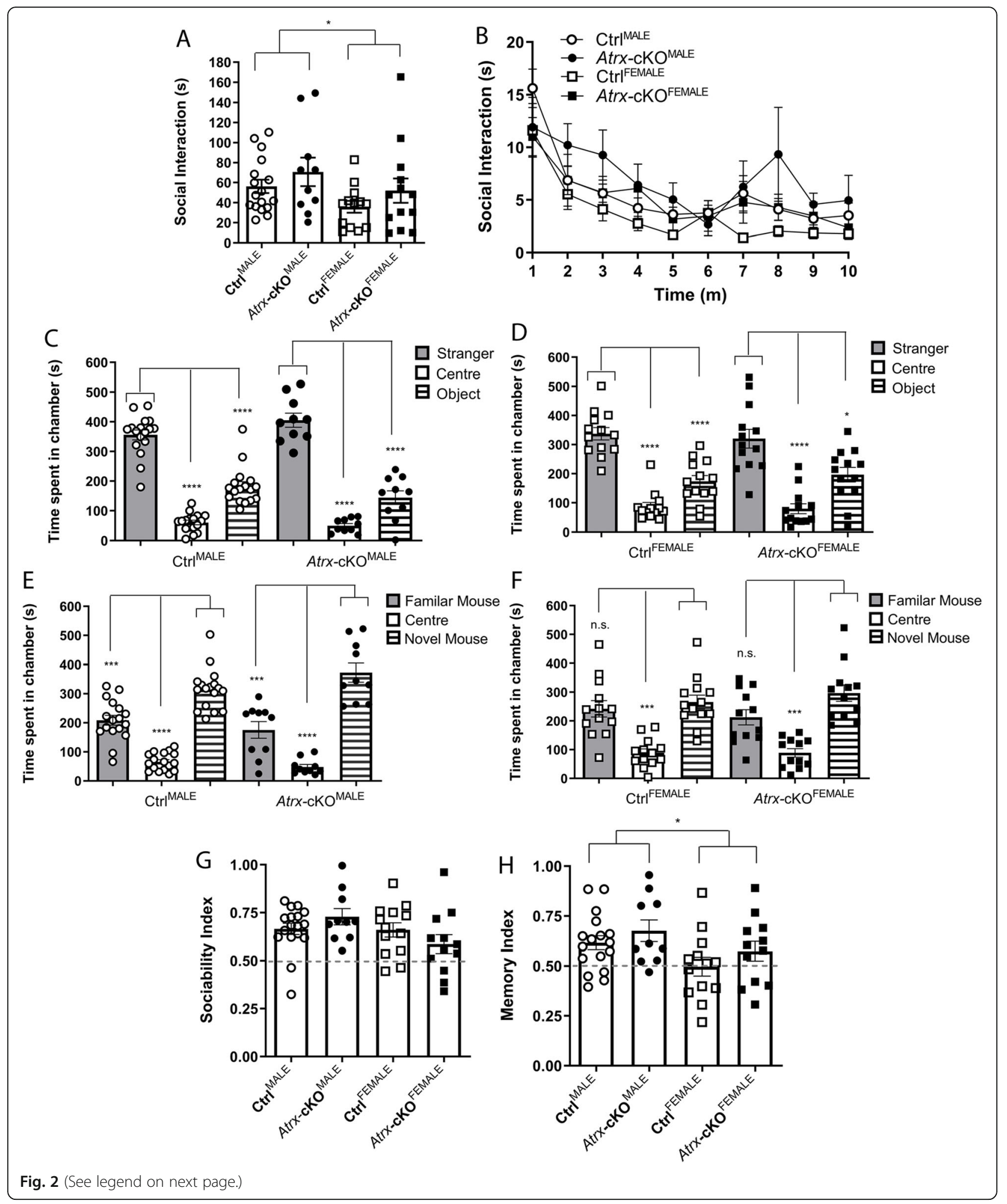




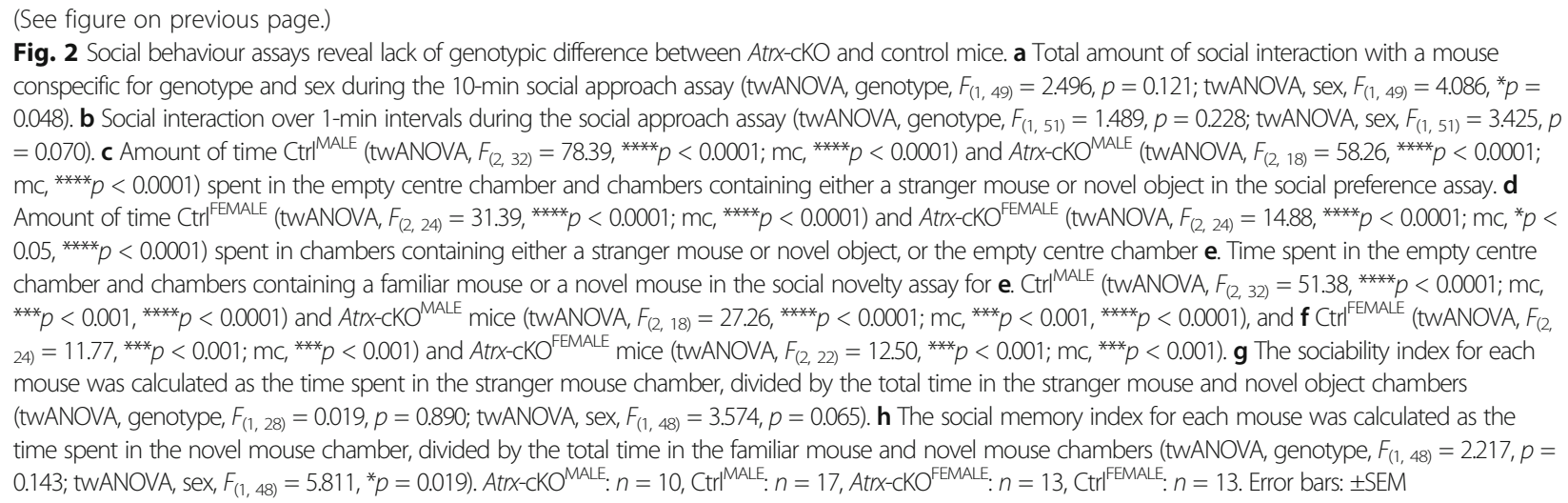

calculated based on the social preference and social novelty results. Atrx-cKO and Ctrl mice did not display genotypic or sex-differences in their sociability indexes (Fig. 2g). Similarly, there was no genotypic difference in social memory indexes, however, male mice $\left(\mathrm{Ctrl}^{\mathrm{MALE}}\right.$ and Atrx-cKO ${ }^{\mathrm{MALE}}$ ) displayed a greater social memory index than female mice $\left(\mathrm{Ctrl}^{\mathrm{FEMALE}}\right.$ and $A$ trx $x$-cKO ${ }^{\mathrm{FE}-}$ MALE) (ANOVA, ${ }^{*} p=0.019$; Fig. 2h). Altogether, these results demonstrate that the loss of Atrx in forebrain excitatory neurons postnatally does not result in social deficits in male and female mice.

Repetitive behaviours are not altered in Atrx-CKO ${ }^{\text {MALE }}$ and Atrx-CKO ${ }^{\text {FEMALE }}$ mice

Previous studies using autism mouse models have demonstrated that the mutant mice often present with repetitive and stereotyped behaviours [27, 34-37]. We tested for the presence of these repetitive and stereotyped behaviours in both $A$ tr $x$-cKO ${ }^{\text {MALE }}$ and Atrx-cKO ${ }^{\text {FEMALE }}$ mice using various tests. The marble-burying assay was used to assess repetitive burying and digging by placing mice in a cage with 12 marbles and recording how many marbles were buried following a 30-min period. Percentage of marbles buried during the marble-burying assay was not significantly different when comparing Atrx-cKO ${ }^{\mathrm{MALE}}$ and Atrx$\mathrm{cKO}^{\mathrm{FEMALE}}$ mice to their respective controls. There also was no difference when comparing between genotypes or sexes. However, the interaction was significantly different between groups, suggesting the loss of Atrx in forebrain excitatory neurons has opposing effects on marble burying when comparing male and female mice (ANOVA, " $p=$ 0.047; Fig. 3a).

We also investigated the presence of repetitive grooming tendencies by misting mice with water to induce grooming behaviours. The total amount of time spent grooming during the 30-min induced self-grooming assay was not significantly different between Atrx-cKO mice and controls, or between sexes (Fig. 3b). When the results of this test were analyzed over 5-min intervals, similarly, there was no difference in the amount of time spent grooming between Atrx-cKO ${ }^{\mathrm{MALE}}$ and $\mathrm{Ctrl}^{\mathrm{MALE}}$ mice (Fig. 3c) or Atrx-cKO ${ }^{\text {FEMALE }}$ and $\mathrm{Ctrl}^{\text {FEMALE }}$ mice (Fig. 3d). Interestingly, results from the open field test show a significant increase in the number of vertical episodes (including rearing and jumping) of female mice (Atrx-cKO ${ }^{\text {FEMALE }}$ and $\mathrm{Ctrl}^{\text {FEMALE }}$ mice) compared to male mice $\left(\right.$ Atr $x$-cKO ${ }^{\text {MALE }}$ and $\mathrm{Ctrl}^{\mathrm{MALE}}$ ) (ANOVA, **:*: $p<0.0001$; Fig. 3e). When these results were analyzed in 10-min intervals, it is apparent that these sexdifferences in vertical episodes occurred primarily within the first $60 \mathrm{~min}$ of the open-field test (multiple comparisons; $" p<0.05,{ }^{* * *} p<0.01$; Fig. 3f). In addition to these sex-differences, there was no genotypic difference between the total vertical episodes or the vertical episodes over time. Similarly, there were no significant differences between Atrx-cKO mice and controls when analyzing vertical episodes. These behavioural analyses suggest that the loss of Atrx in forebrain excitatory neurons postnatally does not result in repetitive or stereotyped behaviours typically associated with autism.

\section{Atrx-CKO ${ }^{\text {MALE }}$ and Atrx-cKO ${ }^{\text {FEMALE }}$ mice display typical startle response to acoustic stimuli}

Previous studies have reported that rodent models with autism-associated genetic mutations can display an exaggerated startle response, or impaired pre-pulse inhibition, to an acoustic stimulus [38-41]. These impairments are associated with deficits in sensory gating and auditory processing often reported in ASD patients [42, 43]. As such, we wanted to investigate if Atrx-cKO mice display hypersensitivity to an acoustic startle stimulus. The pre-pulse inhibition and startle response assay using an acoustic stimulus was performed, as described previously [31]. Mice were placed in a chamber and exposed to 50 trials of an acoustic stimulus $(20 \mathrm{~ms}, 115 \mathrm{db})$. Atr $x$-cKO ${ }^{\mathrm{MALE}}$ and Atrx-cKO ${ }^{\text {FEMALE }}$ mice demonstrated a similar startle response to the acoustic stimuli compared to their respective controls (Fig. 4a, b). Additionally, there was no 


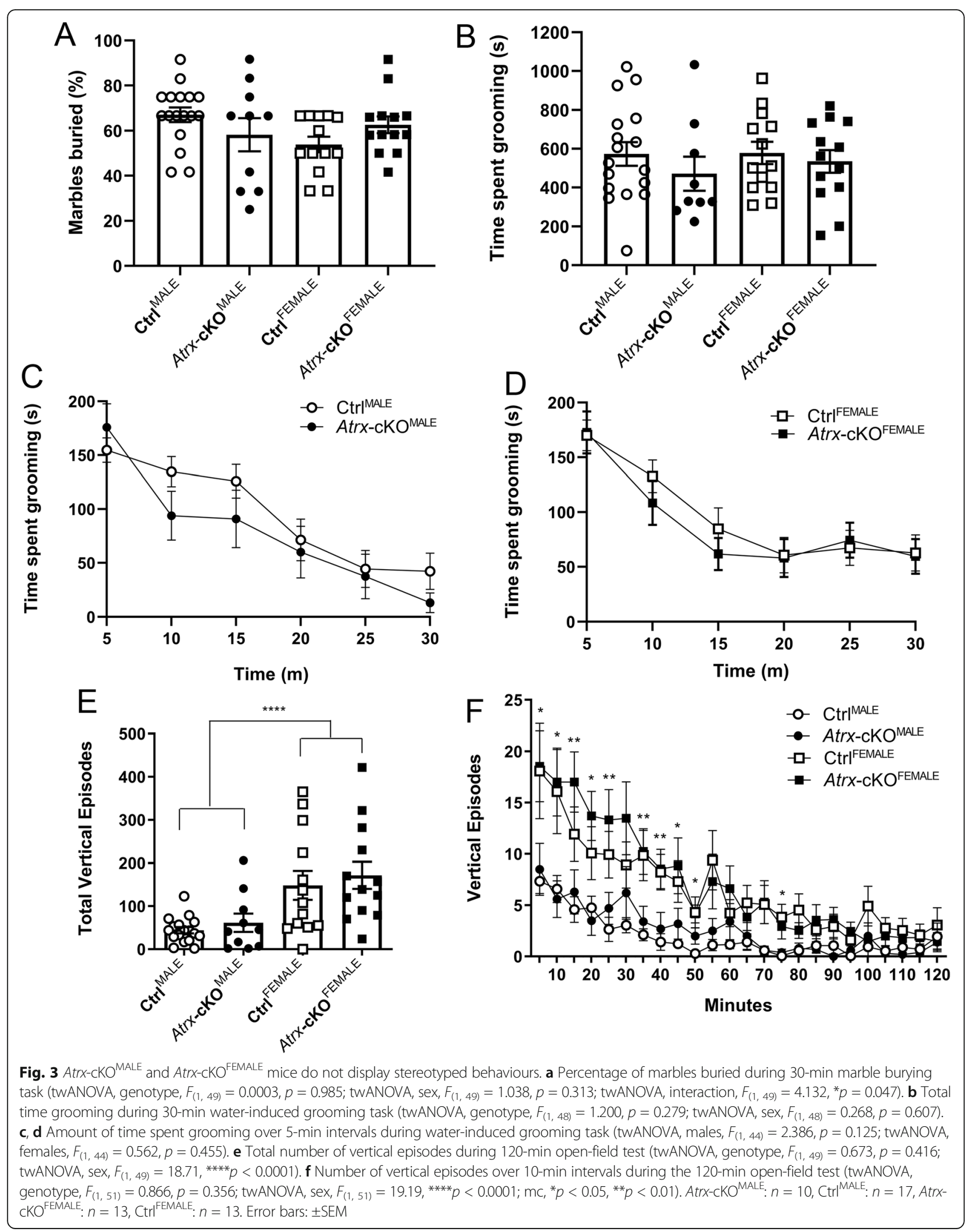




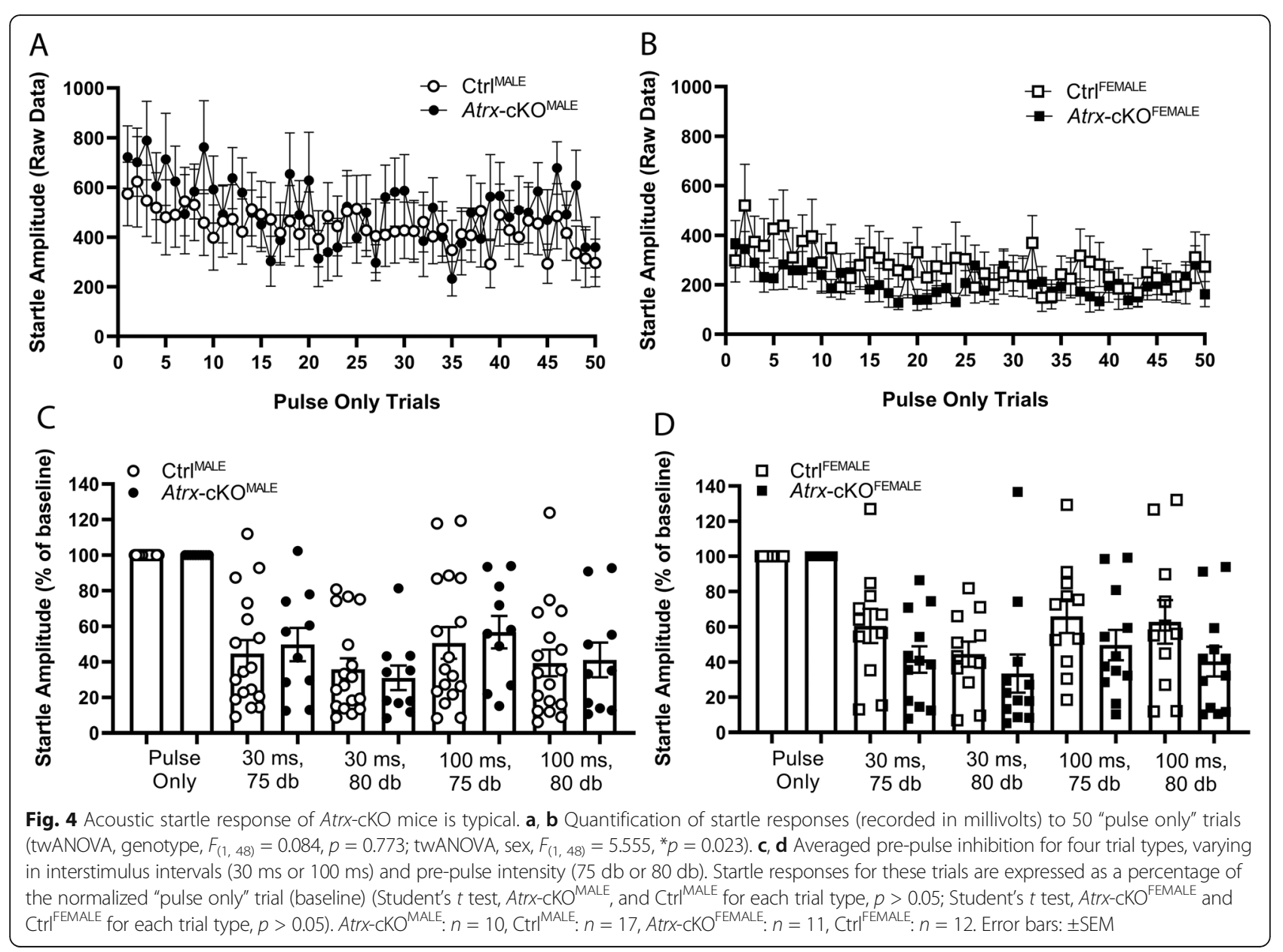

significant difference in startle responses when comparing genotypes (Atrx-cKO vs. Ctrl). Notably, there was a significant increase in the startle response of male mice (Atrx-cKO ${ }^{\text {MALE }}$ and $\mathrm{Ctrl}^{\mathrm{MALE}}$ ) compared to female mice (Atr $x$-cKO ${ }^{\text {FEMALE }}$ and Ctrl ${ }^{\text {FEMALE}}$ ) (ANOVA, " $p<$ $0.023)$. We also performed a set of trials that investigated pre-pulse inhibition to the acoustic stimulus by first exposing mice to a pre-pulse that preceded the acoustic stimulus. These trials varied in the intensity of the pre-pulse ( $75 \mathrm{db}$ or $80 \mathrm{db}$ ), and the amount of time between the pre-pulse and the acoustic "pulse" stimulus (30 ms or $100 \mathrm{~ms}$ ). Additionally, there was one trial that only involved a "pulse" without a pre-pulse. Startle responses to this "pulse-only" trial were used as a baseline, and results from all other trials were expressed as a percentage of this baseline. For all pre-pulse trials, both $A$ tr $x$-cKO ${ }^{\text {MALE }}$ and Atrx-cKO ${ }^{\text {FEMALE }}$ mice did not demonstrate significant difference in their startle responses to the acoustic stimulus compared to the controls (Fig. 4c, d). Overall, these results suggest that the postnatal loss of Atrx in neurons does not result in an exaggerated startle response or impaired pre-pulse inhibition in male or in female mice.

\section{Discussion}

In this study we present an assessment of the effects of a postnatal conditional knockout of the autism susceptibility gene Atrx on autistic-like behaviours in male and female mice. We provide evidence that the postnatal loss of Atrx in forebrain excitatory neurons does not result in social deficits, stereotypies and repetitive behaviours, or sensory gating deficits. We identified differences in olfaction for both male and female mice upon the postnatal conditional loss of Atrx in neurons; however, these differences in olfaction did not impair social behaviours.

Prior to investigating ASD-related behaviours, we first sought to determine whether Atrx-cKO adult mice present with any olfactory differences compared to controls. We performed the odor discrimination and habituation assay to assess if Atrx-cKO mice showed habituation to a repeatedly presented odor and were able to discriminate between a novel odor [25]. By establishing that experimental mice are able to detect and discriminate between odors, results of subsequent social behaviour assays can be more accurately interpreted. Results of the odor habituation and discrimination assay suggest that $A$ trx $x$-cKO $\mathrm{CKALE}^{\mathrm{M}}$ mice have deficits in olfaction, as they spent overall less 
time smelling multiple odors compared to controls. In particular, odor discrimination may be affected in Atrx$\mathrm{cKO}^{\mathrm{MALE}}$ mice as demonstrated by decreased time smelling a novel odor following repeated presentation of another. These differences in olfaction displayed by Atrx$\mathrm{CKO}^{\mathrm{MALE}}$ mice are important to consider when interpreting results from social behaviour assays that require odor discrimination. It is interesting to note that olfaction deficits have been reported in both ASD patients as well as models with autism-associated genetic mutations. In a recent clinical study, nine children with ASD demonstrated impaired olfactory adaption compared to a control group [44]. Additionally, another study reported that mice with haploinsufficiency of the autism-associated gene, T-box, Brain 1 (Tbr 1$)$, displayed impairments in olfactory discrimination [45]. Therefore, these impairments in olfaction, particularly odor discrimination, may be an indication of autistic-like features.

While there was no overall difference in olfaction when we compared Atrx-cKO ${ }^{\text {FEMALE }}$ mice to controls, Atrx-cKO ${ }^{\text {FEMALE }}$ mice demonstrated increased time spent sniffing the cotton swab when presented with the banana odor for the first trial. These results suggest that their odor discrimination may be heightened compared to that of $\mathrm{Ctrl}^{\mathrm{FEMALE}}$. Altogether, although Atrx-cKOMALE and Atrx-cKO ${ }^{\text {FEMALE }}$ mice display differences in olfaction compared to controls, we did not identify any genotypic-differences in social behaviours either in the social approach test or the 3-chamber paradigm. Therefore, any differences in olfaction of Atrx-cKO mice did not result in impairments in social recognition or discrimination based on olfactory cues.

Although there were no identified genotypic effects on the behaviours analyzed here, analysis of the data revealed potential sex-differences in sociability, social novelty, startle response, and anxiety levels. Sex differences in startle amplitude and marble burying have not yet been reported in the literature for the hybrid strain that was used in this study and should be repeated using a replication cohort. However, it is important to note that our experimental approach was not designed to detect differences based on sex, as male and female mice were obtained from different generations of strain hybrids.

The Atrx-cKO ${ }^{\text {FEMALE }}$ mice used in our study experience a complete loss of ATRX expression in forebrain excitatory neurons postnatally. However, in humans, females harbouring ATRX mutations are typically carriers and are asymptomatic due to skewed X-inactivation [12-18]. As such, the clinical relevance of any observed differences we observed in this model are limited. Nevertheless, our model allows the exploration of the basic biology of ATRX function in neurons and the potential effects on the behavioural outcomes. We theorize that the absence of autistic-like phenotypes observed in Atrx-
cKO mice is due to the timing at which Atrx is deleted in forebrain excitatory neurons, which starts at 2-3 weeks of age. ASD is clinically defined as a developmental disorder due to the majority of symptoms becoming apparent in the first few years of life. Therefore, genetic mutations that contribute to autistic phenotypes may need to occur during embryogenesis or be inherited [1, 2 , 4]. Future studies should utilize additional Cre/loxp systems to investigate if the loss of Atrx in differentiated forebrain excitatory neurons during embryogenesis leads to autistic-like behaviours in male and female mice.

\section{Conclusions}

In conclusion, a postnatal conditional knockout of the autism susceptibility gene Atrx did not result in autistic-like behaviours in either male or female mice. Although changes in olfaction were observed in both male and female AtrxcKO mice, these differences did not result in impaired social recognition or discrimination. These findings suggest that the postnatal loss of ATRX is insufficient to cause the subset of autistic behaviours tested here and support the idea that ASD is a developmental disorder where disruptions occur at early stages of brain development.

\section{Abbreviations \\ Atrx: Alpha-thalassemia mental retardation, X-linked; cKO: Conditional knockout; ASD: Autism spectrum disorder; twANOVA: Two-way ANOVA; mc: Multiple comparisons}

\section{Acknowledgements}

We are grateful to Doug Higgs and Richard Gibbons for the Atrx floxed mice, and Vania Prado and Marco Prado for the CaMKII-Cre mice. We are also grateful to Renee Tamming and Radu Gugustea for their aid in mouse husbandry. All behavioural tests were performed at the Robarts Research Institute neurobehavioural core facility.

\section{Authors' contributions}

NMK participated in the design of the study, carried out the experiments and genotyping, analyzed the data, interpreted the results, and wrote the manuscript. NGB contributed to the conception and design of the study, interpretation of data, and writing of the article. All authors read and approved the final manuscript.

\section{Funding}

NMK received paediatrics graduate scholarships from the Department of Paediatrics at Western University. This work was supported by BrainsCAN through the Canada First Research Excellence Fund and by operating funds from the Canadian Institutes for Health Research to NGB (MOP142369).

\section{Availability of data and materials}

All data generated or analysed during this study are included in this published article.

\section{Ethics approval and consent to participate} Not applicable

Consent for publication

Not applicable

Competing interests

The authors declare that they have no competing interests. 


\section{Author details}

'Department of Paediatrics, Schulich School of Medicine and Dentistry, Western University, London, Ontario, Canada. ${ }^{2}$ Department of Anatomy and Cell Biology, Schulich School of Medicine and Dentistry, Western University, London, Ontario, Canada. 'Division of Genetics and Development, Children's Health Research Institute, London, Ontario, Canada. ${ }^{4}$ Department of Oncology, Schulich School of Medicine and Dentistry, Western University, London, Ontario, Canada.

Received: 19 December 2019 Accepted: 4 June 2020

\section{Published online: 24 June 2020}

\section{References}

1. Abrahams BS, Geschwind DH. Advances in autism genetics: On the threshold of a new neurobiology. Nat Rev Genet. 2008;9(5):341-55.

2. Chen JA, Peñagarikano O, Belgard TG, Swarup V, Geschwind DH. The Emerging Picture of Autism Spectrum Disorder: Genetics and Pathology. Annu Rev Pathol Mech Dis. 2015;10(1):111-44.

3. Sanders SJ, Murtha MT, Gupta AR, Murdoch JD, Raubeson MJ, Willsey J, et al. De novo mutations revealed by whole exome sequencing are strongly associated with autism. Nature. 2013;485(7397):237-41.

4. Geschwind DH. Genetics of autism spectrum disorders. Trends Cogn Sci. 2011;15(9):409-16.

5. Yang G, Sau C, Lai W, Cichon J, Li W. Synaptic, transcriptional, and chromatin genes disrupted in autism. Nature. 2014;515(7526):209-15.

6. Loke YJ, Hannan AJ, Craig JM. The role of epigenetic change in autism spectrum disorders. Front Neurol. 2015;6:1-18.

7. Silverman JL, Yang M, Lord C, Crawley JN. Behavioural phenotyping assays for mouse models of autism. Nat Rev Neurosci. 2010;11(7):490-502.

8. Crawley JN. Behavioral Phenotyping Strategies for Mutant Mice. Neuron. 2008;57(6):809-18

9. Gibbons RJ, Picketts DJ, Villard L, Higgs DR. Mutations in a putative global transcriptional regulator cause $X$-linked mental retardation with athalassemia (ATR-X syndrome). Cell. 1995;80(6):837-45.

10. Gibbons RJ, Wada T, Fisher CA, Malik N, Mitson MJ, Steensma DP, et al. Mutations in the chromatin-associated protein ATRX. Hum Mutat. 2008; 29(6):796-802

11. Gibbons R. Alpha thalassaemia-mental retardation, X linked. Orphanet J Rare Dis. 2006;1(1):1-9.

12. Sun M, Paciga JE, Feldman RI, Yuan ZQ, Coppola D, Lu YY, et al. Large-scale discovery of novel genetic causes of developmental disorders. Cancer Res. 2001;61(16):5985-91.

13. Yu TW, Chahrour MH, Coulter ME, Jiralerspong S, Ataman B, Harmin DA, et al. Using whole exome sequencing to identify inherited causes of autism. Neuron. 2013;77(2):259-73.

14. Gong X, Bacchelli E, Blasi F, Toma C, Betancur C, Chaste P, et al. Analysis of $X$ chromosome inactivation in autism spectrum disorders. Am J Med Genet Part B Neuropsychiatr Genet. 2008;147(6):830-5.

15. Brett M, McPherson J, Zang ZJ, Lai A, Tan ES, Ng I, et al. Massively parallel sequencing of patients with intellectual disability, congenital anomalies and/or autism spectrum disorders with a targeted gene panel. PLoS One. 2014:9(4):1-9.

16. Munnich A, Demily C, Frugère L, Duwime C, Malan V, Barcia G, et al. Impact of on-site clinical genetics consultations on diagnostic rate in children and young adults with autism spectrum disorder. Mol Autism. 2019;10(1):1-10.

17. Aspromonte MC, Bellini M, Gasparini A, Carraro M, Bettella E, Polli R, et al. Characterization of intellectual disability and autism comorbidity through gene panel sequencing. Hum Mutat. 2019;40(9):1346-63.

18. Li J, Wang L, Guo H, Shi L, Zhang K, Tang M, et al. Targeted sequencing and functional analysis reveal brain-size-related genes and their networks in autism spectrum disorders. Mol Psychiatry. 2017;22(9):1282-90.

19. Levy MA, Kernohan KD, Jiang Y, Bérubé NG. ATRX promotes gene expression by facilitating transcriptional elongation through guanine-rich coding regions. Hum Mol Genet. 2014;24(7):1824-35.

20. Kernohan KD, Jiang Y, Tremblay DC, Bonvissuto AC, Eubanks JH, Mann MRW, et al. ATRX Partners with Cohesin and MeCP2 and Contributes to Developmental Silencing of Imprinted Genes in the Brain. Dev Cell. 2010; 18(2):191-202

21. Tamming R, Dumeaux V, Langlois L, Ellegood J, Qiu LR, Jiang Y, et al. Atrx deletion in neurons leads to sexually-dimorphic dysregulation of miR-137 and spatial learning and memory deficits. bioRxiv. 2019:1-40.
22. Watson LA, Solomon LA, Li JR, Jiang Y, Edwards M, Shin-Ya K, et al. Atrx deficiency induces telomere dysfunction, endocrine defects, and reduced life span. J Clin Invest. 2013;123(5):2049-63.

23. Bérubé NG, Mangelsdorf $M$, Jagla M, Vanderluit J, Garrick D, Gibbons RJ, et al. The chromatin-remodeling protein ATRX is critical for neuronal survival during corticogenesis. J Clin Invest. 2005;115(2):258-67.

24. Tsien JZ, Chen DF, Gerber D, Tom C, Mercer EH, Anderson DJ, et al. Subregion- and cell type-restricted gene knockout in mouse brain. Cell. 1996:87(7):1317-26.

25. Arbuckle EP, Smith GD, Gomez MC, Lugo JN. Testing for Odor Discrimination and Habituation in Mice. J Vis Exp. 2015;99:1-7.

26. Jamain S, Radyushkin K, Hammerschmidt K, Granon S, Boretius S, Varoqueaux $F$, et al. Reduced social interaction and ultrasonic communication in a mouse model of monogenic heritable autism. Proc Natl Acad Sci. 2008;105(5):1710-5.

27. El-Kordi A, Winkler D, Hammerschmidt K, Kästner A, Krueger D, Ronnenberg A, et al. Development of an autism severity score for mice using Nlgn4 null mutants as a construct-valid model of heritable monogenic autism. Behav Brain Res. 2013;251:41-9.

28. Deacon RMJ. Digging and marble burying in mice: Simple methods for in vivo identification of biological impacts. Nat Protoc. 2006;1(1):122-4.

29. Kalueff AV, Wayne Aldridge J, Laporte JL, Murphy DL, Tuohimaa P. Analyzing grooming microstructure in neurobehavioral experiments. Nat Protoc. 2007; 2(10):2538-44.

30. Tamming RJ, Siu JR, Jiang Y, Prado MAM, Beier F, Bérubé NG. Mosaic expression of Atrx in the mouse central nervous system causes memory deficits. Dis Model Mech. 2017;10(2):119-26.

31. Valsamis B, Schmid S. Habituation and Prepulse Inhibition of Acoustic Startle in Rodents. J Vis Exp. 2011:55:1-10.

32. Tong DL, Chen RG, Lu YL, Li WK, Zhang YF, Lin JK, et al. The critical role of ASD-related gene CNTNAP3 in regulating synaptic development and social behavior in mice. Neurobiol Dis. 2019;130:104486.

33. Courchet V, Roberts AJ, Meyer-Dilhet G, Del Carmine P, Lewis TL, Polleux F, et al. Haploinsufficiency of autism spectrum disorder candidate gene NUAK1 impairs cortical development and behavior in mice. Nat Commun. 2018;9(4289).

34. Nuytens K, Gantois I, Stijnen P, Iscru E, Laeremans A, Serneels L, et al. Haploinsufficiency of the autism candidate gene Neurobeachin induces autism-like behaviors and affects cellular and molecular processes of synaptic plasticity in mice. Neurobiol Dis. 2013;51:144-51.

35. Burrows EL, Laskaris L, Koyama L, Churilov L, Bornstein JC, Hill-Yardin EL, et al. A neuroligin-3 mutation implicated in autism causes abnormal aggression and increases repetitive behavior in mice. Mol Autism. 2015; 6(62).

36. Cheng Y, Wang ZM, Tan W, Wang X, Li Y, Bai B, et al. Partial loss of psychiatric risk gene Mir137 in mice causes repetitive behavior and impairs sociability and learning via increased Pde10a. Nat Neurosci. 2018;21.

37. Jung $H$, Park H, Choi Y, Kang H, Lee E, Kweon H, et al. Sexually dimorphic behavior, neuronal activity, and gene expression in Chd8-mutant mice. Nat Neurosci. 2018;21(9):1218-28.

38. Jiang DY, Wu Z, Forsyth CT, Hu Y, Yee SP, Chen G. GABAergic deficits and schizophrenia-like behaviors in a mouse model carrying patient-derived neuroligin-2 R215H mutation. Mol Brain. 2018;11(1):1-11.

39. Luo J, Norris RH, Gordon SL, Nithianantharajah J. Neurodevelopmental synaptopathies: Insights from behaviour in rodent models of synapse gene mutations. Prog Neuro Psychopharmacol Biol Psychiatry. 2018;84:424-39.

40. Crawley JN. Mouse behavioral assays relevant to the symptoms of autism. Brain Pathol. 2007;17(4):448-59.

41. Scott KE, Schormans AL, Pacoli K, De Oliveira C, Allman BL, Schmid S. Altered auditory processing, filtering, and reactivity in the Cntnap2 knockout rat model for neurodevelopmental disorders. J Neurosci. 2018; 38(40):8588-604.

42. Takahashi $H$, Kamio Y. Acoustic startle response and its modulation in schizophrenia and autism spectrum disorder in Asian subjects. Schizophr Res. 2018;198:16-20.

43. Ebishima K, Takahashi H, Stickley A, Nakahachi T, Sumiyoshi T, Kamio Y. Relationship of the acoustic startle response and its modulation to adaptive and maladaptive behaviors in typically developing children and those with autism spectrum disorders: A pilot study. Front Hum Neurosci. 2019:13:1-6.

44. Kumazaki H, Okamoto M, Yoshimura Y, Ikeda T, Hasegawa C, Saito DN, et al. Brief Report: Odour Awareness in Young Children with Autism Spectrum Disorders. J Autism Dev Disord. 2018;50:1809-15. 
45. Huang TN, Yen TL, Qiu LR, Chuang HC, Lerch JP, Hsueh YP. Haploinsufficiency of autism causative gene Tbr1 impairs olfactory

discrimination and neuronal activation of the olfactory system in mice. Mol Autism. 2019;10(1):1-16.

\section{Publisher's Note}

Springer Nature remains neutral with regard to jurisdictional claims in published maps and institutional affiliations.

Ready to submit your research? Choose BMC and benefit from:

- fast, convenient online submission

- thorough peer review by experienced researchers in your field

- rapid publication on acceptance

- support for research data, including large and complex data types

- gold Open Access which fosters wider collaboration and increased citations

- maximum visibility for your research: over $100 \mathrm{M}$ website views per year

At BMC, research is always in progress.

Learn more biomedcentral.com/submissions 